\title{
Experience in teaching the laser interferometry course in Moscow Power Engineering Institute
}

Bronius Rinkevichius, Alexander Karasik, V. Zubov

Bronius S. Rinkevichius, Alexander Ya. Karasik, V. A. Zubov, "Experience in teaching the laser interferometry course in Moscow Power Engineering Institute," Proc. SPIE 2525, 1995 International Conference on Education in Optics, (13 October 1995); doi: 10.1117/12.224069

Event: SPIE's 1995 International Symposium on Optical Science, Engineering, and Instrumentation, 1995, San Diego, CA, United States 
Experience in teaching the laser interferometry course in Moscow Power Engineering Institute.

$$
\text { B.S.Rinkevichius, A.Ya.Karasik, V.A.Zubov }
$$

V.A.Fabrikant Physics Department Moscow Power Engineering Institute (Technical University) Krasnokazarmennaya, 17, Moscow 111250, Russia

\section{Abstract}

We present a content of a teaching course on the laser interferometry. We consider paragraphs of optical two beams, holographic, speckle interferometry, laser Doppler anemometry, fiber-optic interferometers and optical correlation processing.

\section{Introduction.}

From time to time, an established area of science suddenly revives and rapidly advances as a result of new ideas, better research techniques and improved equipment. An example of this is optic interferometry, which has been transformed by the laser. The conventional distancing techniques have been improved and new ones added, e.g., heterodyne, Doppler, speckle interferometry and interference holography.

Laser interferometry (LI) has now somewhat stabilized as a branch of laser metrology, and so it is opportune now to compile a monograph generalizing current achievements, overviewing the discipline and presenting the principles, technical possibilities, and applications of the method.

The peak of classical interferometry was reached by Michelson, who showed how sensitive it was. Recent advances are as spectacular, viz.,

-the increase in the sensitivity of optical interferometers due to the electronic recording of the interference patterns and their electronic processing;

-the establishing of modulated and heterogeneous interferometry;

-the invention of holographic and speckle interferometry and the application of interference techniques to course surfaces; -the development of laser-Doppler anemometry, which is an interference method for recording the laser spectra scattered by moving particles;

-and, finally, the creation of laser ring interferometry based on the sagnac effect for measuring the rotations of objects.

The techniques all rely on the laser as a source of coherent radiation. The very long coherency distance of the laser may lead to interferometers with distance differences of up to a kilometer. This means that a technique can be applied to a study of seismic activity within the Earth's core and to the search for gravity waves.

classical interferometers using lasers have been created and various topics in quantum and nonlinear interferometry have been 
examined.

At the present time a technique and methods of the laser II find expanding applications in science, engineering and industry. The technique of the LI is used for precise measurements with subwavelength accuracy on vibration setups and at a performance of measurements in the assembly and fabrication of the large industrial products. The courses of the LI occupy a prominent place in teaching courses of different specialities on the cathedra in the honor of V.A.Fabrikant of Moscow Power Engineering Institute. In particular, these materials are proposed for students which are specialized in the field of optics and optoelectronic systems. The questions of the LI are presented for the students of other cathedras as the special course, so a part of the courses of metrology, measurements technique, methods of an optics design. A number of questions, which coincide with scientific directions of cathedra groups, are studied during the process of the carrying out laboratory works. It is necessary to point out the special work of students in scientific groups as Moscow Power Engineering Institute, so in the leading industrial and Academy Institutes :. Physical Lebedev Institute, General Physics Institute, High Temperatures Institute, and Institute of Mechanic Problems.

Next we present the content of the parts of the teaching materials relating to special courses of LI, components of other courses including questions of LI, laboratory works, calculations, teaching and scientific works.

\section{Optical Interference.}

Laser interferometry covers a variety of optical and optoelectronic techniques based on optical interference. classical interferometry deals with the the interference patterns formed by light beams, which yields information about an object interacting with one of the interfering beams; one beam is called the reference beam and the other the object beam. The reference beam should be as ideal in shape as possible in order to facilitate the interpretation of the fringe pattern. The reference beam would be ideal if it had a flat or a spherical wave front. However, classical light sources have low spectral power densities and emit low quality beams. Besides, quality of the intefferometer surfaces appreciably affects the interference pattern.

The situation changed with the advent of lasers, i.e., coherent sources. Basov, Prokhorov, and Townes were awarded the Nobel Prize for their work on quantum electronics and can undoubtedly be called the founders of laser interferometry. Lasers not only improved classical interferometric methods, but also led to new kinds of 2 interferometry, viz., holographic, Doppler, Sagnac and speckle. 2

In holographic interferometry ${ }^{3}$, a wave is first made to produce a hologram and then the interference pattern is ascertained and studied. Holography enables us to implement the differential concept of interferometry with a nonplanar reference wave so as to compensate for imperfections in the optical 
components of the interferometer. Holographic methods yield the interference patterns formed by waves propagating at different periods in time.

Doppler interferometry, also referred to as Laser Doppler Anemometry, yields the interference patterns resulting from laser beams scattered by moving particles. Due to their high spatial coherence laser beams can be focused to a small spot to ensure a high power density within the spot. As a result, the interference pattern originating from the light waves scattered by small particles is bright and can readily be traced by a photodetector.

Speckle interferometry ${ }^{5}$ deals with the interference patterns generated by light waves scattered by rough surfaces.

First, we shall discuss the spatial and temporal properties of light waves. An analysis of the temporal characteristics is confined to the simplest models of monochromatic and quasimonochromatic waves, most important for laser interferometry. The spatial models are confined to plane and spherical waves and also to Gaussian beams.

Further, we shall consider the general regularities of light wave interference, which involve the fringe visibility and the fringe spacing of the interference field, and also describe a three-dimensional interference fields resulting from the superposition of two plane waves, a plane and spherical wave, and two spherical waves.

Unlike the traditional analysis of interference patterns produced by optical devices (a plane-parallel plate, wedge, or Newton rings) this approach enables us to define the general rules governing interference that are inherent in all methods of laser interferometry.

Polarization of the light waves substantially affects the shape of the interference patterns. For this reason, the chapter covers the basic methods of polarization, in particular, the formalism of Jones matrices and the analysis of the basic cases of polarized wave interference.

Laser beam interference has specific features. We shall consider the interference patterns due to Gaussian beams and clarify the conditions governing the generation of traveling interference fringes which result from the interference between monochromatic waves of different frequencies.

In the last section we consider briefly the interference of partially coherent beams and derive, in the general form, formula for beam interference that is suitable for analyzing interference patterns resulting from beams with various characteristics.

\section{Two beams Interferometry.}

Present-day laser interferometers substantially differ from the Michelson interferometer. The implementation of the methods of adaptive optics in interferometry allows to reduce markedly the effects of vibration and noise. The use of the methods of nonlinear optics, feedbach- coupling methods, frequencymodulated semiconductor lasers, and optical-fiber elements open up new possibilities in interferometry. 
Modern laser interferometers can measure linear
displacements, plainness, angles of rotation, an index of refraction, and other parameters. The works enumerated below describe the application of laser interferometers designed to measure the following: linear dimensions of large-size workpieces; temperature coefficient of thermal expansion; gas pressure; free fall acceleration; deformations of the Earth crust; surface contours; refractive indices of gases, liquids and plasma; parameters of vibrations; and angular displacements. The problems of laser interferometry are also dealt with in works.

The modern laser interferometer is a complex opticalelectronic measuring system that includes a laser, an optical unit, a photoelectric transducer, an electronic processor, and software tools. Optimum design of such a measuring system certainly calls for extensive trade-off of the parameters of all its components.

We consider two aspects relating to the interferometric systems, namely, optical signal shaping and processing of phaseand frequency-modulated signals. The methods of measuring the parameters of frequency- and phase-modulated signals are the subject matter of radioelectronics. It is impossible to extend these methods as such into the field of optical interferometry for a number of reasons. One of the reasons is that noise present in signals of laser interferometers is mainly multiplicative in character, whereas in radioelectronic devices the sources of noise are additive.

We consider the principle of a laser interferometer of the homodyne type with photoelectric detection of the fringe shift. The text provides a detailed analysis of the output characteristic of the interferometer, i.e., the output signal as a function of the optical phase shift, examines the interrelation of this characteristic with the main parameters of the laser and optical system, and describes the methods for extending the capabilities of the homodyne interferometer, namely, the phase quadrature methods for determining the sign of the phase difference.

Further, we review the switching interferometers which rely on optical phase-shift modulation to increase their sensitivity. These interferometers can detect extremely small linear displacements down to and including the region of angstroms. Heterodyne interferometers represent a new class of laser interferometers which rely on the internal or external frequency modulation of laser beams to perform measurements with the aid of traveling interference fringes.

In the later section the discussion centers on the features of operation and the field of application of active interferometers which form wide class of laser interferometers where the laser resonator serves as a constituent of the interferometric system.

What is important for gaining a better insight into the operation of laser interferometers is the analysis of the methods of measuring the phase of an electric signal that carries information on the phase difference of laser beams. The text 
provides coverage of both the methods of measuring the fringes of the integral order of interference and the methods of measuring a fraction of the fringe.

\section{Laser-Doppler Anemometry.}

In an article "On the color of Binary stars and some Heavenly Bodies", published in 1842, Doppler considered how the movement of a wave source (transmitter) or the receiver (observer) of the waves affected the observed frequency, i.e., its pitch or color. Doppler did not distinguished between light and sound waves and considered the frequency change to be independent of the type of wave. His ideas were the subject of a lively discussion for many years, and it was not until about 30 years later that were accepted after the experimental verification of the change in frequency of both sound and optical waves.

The Doppler effect is widely used in various measurement techniques for solving inverse problems, namely, determining the speed of an object from the measured shift in the frequency of radiation emitted or scatted by an object. It is crucial in astrophysics to measure the speeds of cosmic objects from line shifts in the spectra they emit; in radar to measure the speed of aircraft; in sonar to detect underwater vehicles; and in plasma physics to estimate the temperature of plasma.

In astrophysics and plasma physics, the measurements are indirect as the parameter desired has to be derived from the actual measurement, e.g., the shift in a spectral line is measured in the radiation from a moving source.

Radar and sonar directly measure using a harmonic source and a unit to find the Doppler shift of the scattered or reflected radiation.

With the advent of coherent light sources the Doppler effect was extensively employed in laser measurements of the velocities of the liquid and gas flows and diffuse-scattering surfaces. This work began with the publication of by Yeh and commins in 1964, who were the first to consider the potential of the photomixing method for measuring the Doppler frequency shift in scattered laser radiation.

Since then, much work has been done to harness lasers to measure the velocities of laminar and turbulent flows of liquids and gases. Thus a new scientific discipline has emerged due to the efforts of many scientific associations, resulting in the theory of flow velocity measurement, a large variety of optical systems for laser measurements, electronic Doppler signal processing, and methods for solving a wide range of aerohydrodynamic problems. The large number of monographs, such as, are indicative of the progress in the field.

We consider the general principles of laser- Doppler anemometers designed to measure the velocities of liquid and gas flows. Unlike sound and radio frequencies, light frequencies cannot be measured directly, and therefore LDA methods require the measurement of the frequency difference between either the scattered and probing beam or two scattered waves. 
Further, we consider laser beam scattering by small particles and various types of scattering: Rayleigh, RayleighGans, Mie scattering, and geometric optical scattering. Particular attention is given to the polarization and phase characteristics of scattered radiation, which are very important for interference effects. The interference patterns produced by oncoming laser beams differ from the interference pattern resulting from scattered light, the principal feature being the variation in the amplitude and phase of the scattered beam with the angle of scattering. In fact, we deal with a speckled structure when laser radiation is scattered by moving particles and its structural parameters recorded.

Two paragraphs the shaping an output Doppler signal by a light sensor as it responds to an optical signal from a nonstationary interference pattern. The parameters of the Doppler signal depend on the laser, optical transmission and receiving systems, light sensor, and the particles and flows involved. Because of the large variety of scattering particles and different flow conditions, the actual Doppler signal is a random signal with random amplitude, phase, frequency, and duration.

Special section presents information on various ways of measuring two components of the velocity vector of a liquid or a gas flow. The measurement of a particle velocity vector requires three independent interference patterns to be measured and the scattered light from each of the pattern must be detected separately.

Finally, we consider various methods for measuring the parameters of Doppler signals, which help define the mean velocity and the degree of turbulence of a flow. This section also describes electronic processors, namely, analyzers of a Doppler signal spectrum, tracking systems, and discrete counting systems.

\section{Fiber-optic Interferometers.}

We describe the basic concepts of interferometers, which use fiber waveguides for channeling light beams. Fiber optics as a logical extension of physical optics has evolved into an individual field of science and engineering that deals with a wide range of problems concerning the research, development, and utilization of fiber guides in various optical devices.

Last years have seen an accelerated trend toward the use of fibers for the sensors of acoustic, thermal, magnetic, gravitational and other fields, which open up new opportunities for controlling these fields by means of the phase, intensity, and polarization state of the laser beam transmitted through fiber guides. The optical fiber sensors designed to respond to changes in the phase of the laser beam down to $10^{-6}-10^{-7}$ rad should be built on the basis of adequate optical systems of interferometers. The optical systems with fiber display a number of new features owing to specific propagation of light through optical fibers.

We focus our attention on the basic wave transmission and polarization characteristics of fiber guides, which are of 
primary importance for the designer of interferometric sensors, consider design features of fibers and describe the basic fiber components of all-fiber interferometers. Since the theoretical concepts of interferometry and classical interferometer arrangements are given detailed treatment in the previous treatments, our principal concern will be the specific aspects relating to fiber-optic interferometers.

We briefly examine the theoretical aspects of propagation of waves in fiber guides and their polarization and describe some optical arrangements of the known interferometers, placing emphasis on performance of the interferometers as high-response sensors. Primary attention is given to a fiber ring interferometer which is the rotation sensor using the sagnac effect. Unlike the optical fiber Mach-Zehnder and Michelson interferometers, in which external fields modulate the refractive index or birefringence of fibers, the fiber-ring sagnac interferometer does not depend on fiber guide properties in a first approximation. Analysis of the effect of various fields on the light phase in the ring interferometer thus enables us to reveal most fully the behavior of other interferometers.

\section{Holographic Interferometry.}

The use of holographic methods in interferometry has opened up new ways for the development of this field of science and engineering.

The principle of holographic interferometry consists in the following. Illuminating a hologram with the coherent light beam reconstructs the image of an object. If it is possible to obtain two or more similar waves representative of the object images at different moments of time, these waves will interfere and produce the fringe pattern which can provide information on the varying object.

In comparison with the methods of classical interferometry, the methods of holographic interferometry offer a number of possibilities briefly outlined below.

1. On a holographic plate there can be recorded the waves scattered from an object at different moments of time. Since at the stage of image reconstruction these waves reproduce simultaneously, they interfere with each other so that holographic interferometry yields the interference pattern yields, which existed at different moments of time.

2 . Image recording can be made at different wavelengths. The light used for reconstruction has merely one wavelength, so that interference of the waves permits observing the fields at different wavelengths.

3. At the stage of recording, the holographic interferometry system operates as a single-channel recording arrangement because the optical way for shaping the light fields from the object has the same effect on the system in all the stages of the object.

4. If the object surface structure remains invariable, it has no effect on the interference pattern reproduces the changes in the object. For this reason, the methods of holographic interferometry are suitable for investigation of both transparent 
and diffuse objects.

5. In the process of recording and reconstruction of the image of an object, the system can produce the waves corresponding to various directions of light propagation from the object within the hologram aperture. This offers the possibility of observing the interference pattern in different directions, thus obtaining a larger amount of information on the distribution and changes of optical inhomogeneities in the object.

6. Holographic interferometry can be used to implement a differential method for reproducing variations in the characteristics of an object over any preset interval of time.

In practice, decision is taken to apply one method or another depending on the tasks involved. These can be the methods of double-exposure, time-average or real-time holographic interrferometry, the method of stroboscopic illumination and the method with changing the conditions of object illumination and recording conditions.

The methods of holographic interferometry can handle a number of practical problems which are impossible or very difficult to solve by other methods. For example, they can be used to analyze changes in the structure of transparent objects such as plasma or glass, plastic and crystalline elements, to investigate the object deformations or vibrations and to study the time variation of the light field from an object.

We consider the basic principles of holography and holographic interferometry. Much attention will be given to new trends in this field, which relate to the use of nonstationary-wave reference beams. Analysis of the methods mentioned above will be undertaken in the framework of physical optics covering the phenomena of interference between the stationary and time-dependent fields and the phenomena of diffraction of scalar fields. A more detailed presentation of these aspects can be found in the books of physical optics and holography.

\section{Speckle Interferometry.}

If an object with diffuse surface is illuminated by coherent light, or if its image is formed with the aid of an optical system, there appears a pattern of small light and dark spots or "speckles". This phenomenon owes its existence to the interference of light waves scattered by this surface. Any image produced by this light is a random interference pattern, because a diffusely scattering surface is characterized by random variations of phase in light scattered when passing from one point to another. The observed grain pattern received the name "speckle pattern". Typical optical speckle patterns are given in the book by Francon. A statistical theory of speckle patterns may be found in fundamental papers of Dainty. Those grain speckle patterns can be observed by illuminating diffuse objects by coherent laser light. Under ordinary conditions such a pattern may be considered as a noise. However, speckles may be sometimes used for data processing and for measuring displacements, deformations, and rotations of diffuse objects or their parts. 
The fundamentals to such applications of speckles were laid in the classical paper by Burch and Tokarski. Later on methods for using speckle patterns developed intensely with a host of researchers involved. Advantages of the speckle- based methods are a simple optical system toleranced to the stability and vibration-resistance of the installations, a simplicity of analyzing experimental data, and adjustable sensitivity.

\section{Optical Correlation Processing.}

Two-beam interferometers have proved to be useful for the correlation of optical information presented on transparencies to extract identified signals in noise. This type of correlator offers a number of advantages, for example, using images of objects rather than specially prepared filters. Differently arranged optical systems allow the correlation of the transparency patterns in one arm and the sun or difference of the fields due to these patterns in the other arm.

The two-beam configuration allows for an accurate equalizing of optical path lengths in both arms and for reliable matching of spatial structures of the two light beams. In is tolerable to incoherence of the illumination and affords coherent processing with incoherent illumination sources. Two independent channels enable different additional processing to be performed in each channel. In particular, both images of objects and their Fourier transforms can present the information to be processed. Representation of the information for one channel can differ from that for the other. Measuring independently the output signals from each channel provides normalizing the result and finally, the absolute value of the correlation functions.

\section{Acknowledgments.}

Authors wish to thank co-workers of V.A.Fabrikant Physics cathedra E.I.Zubarev, A.V.Stepanov and V.V.Tolkachev for the help in a creation of a laser laboratory.

\section{References.}

1. M.Born and E.Wolf Principles of Optics, 6-th ed., New York, Pergamon Press, 1980.

2. A.Ya.Karasik, B.S.Rinkevichius, V.A.Zubov "Laser Interferometry Principles". Tokyo, London: CRC Press, Mir. Publishers, Moscow, 1995.

3. R.J.Coller, C.B.Burckhardt, L.H.Lin "Optical Holography" New York, Academic Press, 1971.

4. Yu.N.Dubnishchev and B.S.Rinkevichius "Methods for LaserDoppler Anemometry", Moscow, Nauka 1982 (in Russian).

5. J.C.Dainty "The statistics of Speckle Patterns" In: Progress in Optics, ed.by E.Wolf, Amsterdam, Norht-Holland, v.14, pp.3-46, 1976. 